\title{
Del finalismo comunista al fin del comunismo
}

Manuel Pastor

Me propongo en este ensayo una reflexión escéptica sobre las determinantes ideológicas del Pez Cornudo. El escepticismo, como señalara Hegel en el capítulo sobre la Autoconciencia (de su obra Fenomenología del Espíritu, 1807) es la realización de aquello de que el estoicismo -primera manifestación consciente de la libertad de la Autoconciencia - era solamente el concepto: "el pensamiento deviene el pensar completo que destruye el ser del mundo múltiplemente determinado, y la negatividad de la autoconciencia libre se convierte, ante esta múltiple configuración de la vida, en negatividad real" (1).

Son la independencia del pensamiento (estoicismo) y la conciencia crítica (escepticismo) quienes encuentran su verdad en aquella figura llamada autoconciencia desventurada que "constitúye el reverso y el complemento de la conciencia dentro de sí perfectamente feliz, de la conciencia cómica" (2). Y, a propósito de comicidad, explicaré que lo del Pez Cornudo era una expresión, una especie de broma, que usaban en sus tertulias algunos intelectuales marxistas críticos (Korsch, Benjamin, Brecht) al referirse al sistema comunista bajo Stalin, aunque seguían pensando que, a pesar de todo, un pez cornudo èra mejor que un pez sin cuernos...

Contrariamente a lo que piensan Samuel P. Huntington y James Atlas (3), la moda del "finalismo" no es tan reciente, y desde luego no es Francis Fukuyama, con su brillante ensayo ¿Fin de la Historia? (4), el responsable. Hace casi treinta años ya señalaba agudamente el politólogo español Jesús Fueyo qưe "los 
dos grandes temas que Hegel deja abiertos a la crítica ulterior de tan impresionante impacto social y político, cuales son el fin de la Historia y la muerte de Dios, son como el canto del cisne del racionalismo europeo" (5).

La muerte de Dios como presupuesto del finalismo comunista en Marx y Engels y de los concomitantes totalitarismos ulteriores, aunque fuera anunciado angustiosamente por Nietzsche en el umbral del siglo $\mathrm{XX}$, gozaba ya de una larga tradición desde el maestro Eckart en la teología negativa germano-protestante de Lutero a Hegel. (6) "Esta muerte -escribirá el filósofo prusiano- es el sentimiento doloroso de la conciencia desventurada de que Dios mismo ha muerto. Esta dura expresión es la simple expresión del simple saber de sí mismo más íntimo, el retorno de la conciencia a las profundidades de la noche del yo=yo, que no diferencia ni sabe ya nada, fuera de ella" (7). El tópico, incoado literal y conceptualmente en la filosofia hegeliana, preside todo el pensamiento secularizado y radical que desde principios del siglo XIX conduce al comunismo (Marx y Engels) y al nihilismo (Stirner y Nietzche), así como toda la reacción católica contrarrevolucionaria (Bonald, Maistre, Donoso Cortés y Carl Schmitt) (8).

El finalismo comunista, que es el objeto de este ensayo, prescindiendo de los precedentes utópicos, tiene su primera expresión "científica" en el Manifiesto Comunista (1848) de Marx y Engels: "La burguesía - proclaman- produce, ante todo, sus propios sepultureros. Su hundimiento y la victoria del proletariado son igualmente inevitables... El rasgo distintivo del comunismo no es la abolición de la propiedad en general, sino la abolición de la propiedad burguesa. Pero la propiedad privada actual, la propiedad burguesa, es la última y más acabada expresión del modo de producción y de apropiación de lo producido basado en los antagonismos de clase, en la explotación de los unos por los otros. En este sentido los comunistas pueden resumir su teoría en esta fórmula única: abolición de la propiedad privada" (9). Resumiendo, la desaparición de la propiedad privada terminará con la explotación económica y la dominación política. La revolución comunista, por tanto, creará las condiciones para la desaparición del poder político: el Estado se extinguirá, y "surgirá una asociación en que el libre desenvolvimiento de cada uno será la condición del libre desenvolvimiento de todos (10). El círculo se cierra, el largo movimiento desde la utopía a la ciencia retorna a la utopía: fin de la propiedad privada, fin de las clases sociales, fin del capitalismo, fin del Estado y fin de Dios. El anunciado fin de la Historia de Hegel se traviste en Anti-Historia. La muerte de Dios, que lleva incoada la muerte del Hombre, proporciona los fundamentos de la utopía totalitaria, pero el comunismo, el socialismo real, incapaz de 
sintonizar con los procesos históricos reales, pronto mostrará su faz auténtica y monstruosa: el Pez Cornudo.

Con el inicio del primer experimento comunista de la historia, tras el golpe bolchevique de octubre de 1917 , se pondrá de manifiesto la dificultad inherente esencial, que una marxista crítica y sensible como Rosa Luxemburg percibirá antes de que transcurra un año: "Lo negativo, la destrucción, sí se puede decretar; la construcción, lo positivo, NO. Tierra virgen. Miles de problemas. Sólo la experiencia está en condiciones de corregir y de abrir nuevos caminos. Sólo una vida llena de fermentos, sin impedimentos, imágina miles de formas nuevas, improvisa, libera una fuerza creadora, corrige espontáneamente sus pasos en falso... Toda la masa del pueblo debe participar; si no, el socialismo es decretado, autorizado desde la mesa por una docena de intelectuales" (11). Crítica impecable que, por cierto, no tuvo en cuenta ella misma más tarde al encabezar la-insurrección espartaquista en Alemania, y que transluce la idea de un socialismo irreal.

El socialismo real parece, lógicamente, la única alternativa al socialismo irreal. El erudito trabajo de Rudolf Bahro (La alternativa. Contribución a la crítica del socialismo realmente existente, 1977) no nos aclara el origen de la expresión, generalizada y asumida por los propios comunistas durante la etapa neo-stalinista, desde Breznev hasta Gorbachov. Mi impresión es que tiene su origen en los debates ideológicos de 1924-1926 acerca del "socialismo en un solo país". En el folleto Los fundamentos del leninismo (mayo de 1924) Stalin sostiene que la perspectiva de la revolución mundial -la acción conjunta de los proletarios de todos los países adelantados o, por lo menos, de la mayoría de ellos-, "ya no corresponde a la realidad" (12). La perspectiva real, se infiere, es la del socialismo en un solo país. La afirmación del socialismo real frente a otras consideraciones abstractas se infiere asimismo del texto de Grigori Zinoviev, El leninismo (escrito en 1924): "Afirmando: el triunfo del socialismo en un solo país, estamos diciendo que los demás países continúan aún siendo burgueses y que el país socialista del que estamos hablando se halla cercado por estados burgueses. No se trata de un país abstracto" (13). En diciembre de ese mismo año, como prefacio a su libro El camino de octubre, Stalin escribirá: "la teoría de la imposibilidad de la victoria del socialismo en un solo país; ha resultado ser una teoría artificial, una teoría no viable" (14). Finalmente, en Cuestiones del leninismo (enero de 1925) el dictador soviético se propone cerrar la discusión, aclarando dos cuestiones distintas: "la cuestión de la posibilidad de llevar a cabo la edificáción del socialismo con las fuerzas de un solo país, cuestión a la que hay que dar una respuesta afirmativa, y la cuestión de si un 
país con dictadura del proletariado puede considerarse completamente garantizado contra la intervención y, por tanto, contra la restauración del viejo régimen, sin una revolución victoriosa en otros países, cuestión a la que hay que dar una respuesta negativa" (15).

En 1936 (inicio de los procesos de Moscú, promulgación de la nueva constitución soviética que sanciona el Estado socialista), cuando Trotsky publica su denuncia anti-stalinista $L a$ revolución traicionada, confiando, empero, en su regeneración a través de la revolución permanente (interna e internacional), el escritor norteamericano Max Eastman (uno de los primeros comunistas occidentales y, probablemente, el primer ex-comunista) concluye un ensayo titulado The End of Socialism in Russia (16) que constituye, por lo que a mí se me alcanza, la primera predicción del fin del comunismo, y, en cierto modo, el inicio de la moda "finalista" (de signo contrario al finalismo comunista) que llega hasta nuestros días: fin del comunismo, fin de las ideologías (A. Camus, R. Aron, D. Bell, E. Shils, ete.), fin de la Historia (A. Kojève, F. Fukuyama) (17). Tras ofrecernos en 1939 el primer análisis comparado nazi-8oviético del Totalitarismo (18), Max Eastman sostendrá que, aparte de la realidad socialista en la URSS, no existe otro socialismo, y que el socialismo democrático es imposible (19). Cuando Eastman afirma - con palabras que impresionarán al economista Friedrich $\mathrm{A}$. Hayek, patriarca del liberalismo conservador de nuestro tiempo- que "el stalinismo es socialismo, en el sentido de ser el acompañamiento político inevitable, aunque imprevisto, de la nacionalización y la colectivización que ha adoptado como parte de su plan para erigir una sociedad sin clases" (20), el escritor norteamericano confiere a la expresión socialismo real una precisión objetiva, al margen de las especulaciones ideológicas. Es el mismo significado que, recientemente, le atribuye Jan S. Prybyla, en uno de los primeros análisis teóricos que se han publicado acerca de la transición del comunismo al capitalismo: "I use the word socialism to denote the system -otherwise known as real, existing socialism - that has actually been applied in this century by ruling communist parties all over the world. I restrict the term to centrally planned, administrative, command economies of which the Soviet Union from Lenin to Gorbachev is an excellent example" (21). El autor nos aclara que cuando él habla de socialismo no se refiere a los significados que le dan algunos intelectuales académicos, los clérigos mesiánicos del Tercer Mundo, o los ideológicos visionarios-utópicos. Prybyla evoca oportunamente las palabras de Hayek en uno de sus últimos trabajos, The Fatal Conceit: The Errors of Socialism (1989), al señalar agudamente que el declive y fin del comunismo se está produciendo principalmente en los países donde realmente se había 
implementado, lo cual facilita la desilusión respecto a la utopía, pero sigue viviendo en el corazón de quienes no han experimentado sus efectos reales: "among Western intellectuals and among the poor ... in the Third World... In the latter, liberation theology may fuse with nationalism to produce a powerful new religion with disastrous consequences for people already in dire economic straits" (22).

El también economista y, como su maestro Hayek, premio Nobel, James Buchanan, ha querido emular el nietzscheano anuncio de la muerte de Dios con el no menos dramático el socialismo ha muerto: "a statament that seems much less shocking than Nietzsche's because it is being heard throughout the world in 1990. In a very real sense, the loss of faith in socialism is more dramatic than the loss of faith in God, because the god that was socialism took on forms that were directly observable. There were no continuing unknowns waiting to be revealed only in another life, and the promised realization of the socialist ideal could not be infinitely postponed. Socialism promised quite specific results; it did not deliver" (23).

Todos los autores aquí citados (Eastman, Hayek, Buchanan, Prybyla) desde una perspectiva demoliberal coinciden en identificar las causas del fin del comunismo en dos errores interrelacionados: uno material (la abolición de la propiedad privada) y otro intelectual (la "abolición" del mercado), ambos expresivos de una ignorancia acerca de la naturaleza humana y de la naturaleza social. Limitándonos aquí al error intelectual, el comunismo o socialismo real ha sido el resultado tangible de una pertinaz obsesión. (neurosis ideológica, la llamaremos siguiendo a A. Koestler, superstición la denomina Hayek, impostura y bluff según K. Popper) (24) de ciertos intelectuales-ideológicos que, desde un enfoque materialista/determinista de la Fistoria (no exclusivamente marxista) han negado sistemáticamente la autonomía de la conciencia individual (¿hay otra?) y la posibilidad de que las ideas tengan consecuencias. El revisionismo, a partir de Eduard Bernstein, lo intuyó, pero el movimiento socialista internacional no ha sido capaz, hasta la fecha, de ser plenamente consecuente con tal percepción. Aunque es cierto, como ha señalado Ramón Cotarelo, que "tras haber conocido momentos de esplendor, la izquierda se bate en retirada. Los comunistas quisieran ser socialistas: democráticos y los socialistas democráticos, demócratas a secas" (25).

Prybyla ha descrito correctamente; a mi juicio, tal error intelectual que, en sintesis, consiste en pensar: a) que alguien situado en el centro o vértice de una organización jerárquica, deliberadamente construida, pueda obtener y procesar toda la información procedente de numerosas y dispersas fuentes; b) que tal información sea mejor que la obtenida a través del proceso del mercado; c) que tal 
información pueda ser utilizada para determinar fines mandatorios, sustitutivos y superiores a los que emergen de las preferencias y elecciones populares. Tales asunciones, efectivamente, no comprenden la naturaleza específica de un conocimiento disperso, autodesarrollado en el proceso del mercado. Desde el punto de vista psicológico y moral el socialismo supone - suposición arrogante y falaz-que los individuos humanos no saben lo que quieren, y por tanto, deben ser aleccionados por alguna "inteligencia superior", con privilegio epistemológico y especial capacidad para comprender la racionalidad social. Como consecuencia de lo anterior, aparece el problema de los incentivos, de manera que, al final, el único medio de reconciliar las preferencias de los individuos con las de los planificadores es la coerción: la fuerza, normalmente acompañada del miedo, que da como resultado el sistema de terror y el universo concentracionario (26).

Quizás no sea ésta la ocasión para detenernos en la fenomenología del post-comunismo y del post-socialismo. Existe ya una importante, aunque no exhaustiva, literatura sobre el tema (27). En las sucesivas fases que Zbigniew Brze: zinski describe para la transición del comunismo al capitalismo (I. Totalitarismo comunista; II. Autoritarismo comunista; III. Autoritarismo post-comunista; y IV. Pluralismo post-comunista) (28) es previsible la supervivencia de estructuras ideológicas comunistas o filocomunistas, que nos permiten pensar en un proceso no irreversible. El riesgo o peligro de regresión resulta evidente en algunas sociedades de la antigua Unión Soviética y del Este de Europa. Por otra parte, la crisis del comunismo no significa la desaparición inmediata del mismo como sistema de dominación sobre un tercio de la población del Planeta, aproximadamente, en países como Afganistán, Albania, Angola, Camboya, China, Congo, Corea del Norte, Cuba, Laos, Mongolia, Mozambique, Vietnam, Yemen, Zimbabue... (29). Pero, desde el punto de vista ideológico, el fin del comunismo, como percepción de la conciencia demoliberal en este fin de siglo-fin de milenio, no significará, desde luego, el fin de las ilusiones ideológico-utópicas de ciertos intelectuales en Occidente, que, como señalará Hayek, no han experimentado precisamente los efectos del socialismo real. Las ya clásicas tesis de Ortega y Benda sobre la responsabilidad y traición de los intelectuales siguen plenamente vigentes (30). El comunismo seguirá siendo, en la afortunada expresión del Raymond Aron, "el opio de los intelectuales" (31).

Por otra parte, la "tentación totalitaria" continuará constituyendo un serio peligro en determinados híbridos ideológicos como el marxista-cristiano de la Teología de la Liberación y el marxista-islámico de ciertas dictaduras y movimientos árabes, tradicionalmente tributarios del comunismo, a los que se puede 
aplicar, parafraseando a Revel, el calificativo de pidgin-comunismo, una especie de potaje ideológico de postulados marxistas-leninistas e izquierdistas aderezados con ingredientes "tercermundistas" (32). Finalmente, esa misma "tentación totalitaria" puede persistir o reaparecer en el seno de algunos partidos socialistas de Occidente si se obstinan en no admitir que la única alternativa efectiva al socialismo real es un sistema de economía de mercado y democracia política (en un sentido formal -Estado de Derecho—y con una cultura auténticamente participativa), ya que, según el criterio general establecido por Kornai, "la frecuencia y la intensidad de la intervención burocrática en el proceso del mercado (económico y político) tiene cierto punto crítico. Una vez que se ha rebasado ese punto crítico, el mercado queda estrangulado y dominado por la regulación burocrática" (33). Criterio que puede ser aplicado, inversamente, en los futuros análisis sobre la intervención de las instituciones del mercado en los sistemas burocrático-colectivistas que están experimentando el fin del comunismo. 
1. G. W. F. Hegel, Fenomenología del Espíritu, FCE, México, DF, 1978, 1p. 122 y 124-125.

2. Ibidem, p. 435.

3. S.P. Huntington, "No Exit. The Errors of Endism", The National Interest, New York, Fall 1989. J. Allas, "Cómo terminar con la historia (La moda finalista)", publicado originalmente en New York Times Magazine, en Diario 16, 26 de noviembre de 1989.

4. F. Fukuyamu, "The End of History?", The National Interest, New York, Summer 1989; y "A Reply to My Critics", The National Interest, New York, Winter 1989-90.

5. J. Fueyo, "El fin de la política moderna", en Estudios de Teoría Politica, IEP, Madrid, 1968, p. 376.

6. B. H. Levy, El testamento de Dios, El Cid, Buenos Aires, 1980, pp. 40 y 50 , donde el autor nos ofrece las referencias al concepto de la muerte de Dios en los escritos del maestro Eckart, de Lutero, e, incluso, en Pascal.

7. Hegel, ob. cit., p. 455.

8. Después de Hegel y antes de Marx y Nietzsche, la expresión Dios ha muerto aparece en la obra de Max Stirner, El Unico y su propiedad (1844): eito la versión en inglés, The Ego and His Own, Harper \& Row, New York, 1971, p. 109. La reacción católica contra el pensamiento revolucionario que se fundamenta en el ateísmo y el agnosticismo, se inspira en Burke, y sus primeros exponentes son Joseph de Maistre (Ensayo sobre el principio generador de las constituciones políticas, 1808-1809, Gateway Ed., Chicago, 1967, pp. 84-85; y Las veladas de San Petersburgo, 1809, Espasa-Calpe, Madrid, 1966, p. 190) y Louis de Bonald (Essai analytique sur les lois naturelles de l'ordre social ou du pouvoir..., París, 1817). Juan Donoso Cortés en su Discurso sobre la Dictadura (1849) establece, por primera vez en el pensamiento contemporáneo, los efectos concomitantes del ateísmo sobre la dictadura totalitaria (Obras Completas, vol. II, Ed. Carlos Valverde, BAC, Madrid, 1970, p. 316). Carl Schmitt ha constatado que "las afirmaciones Dios ha muerto y el poder es en sí malo proceden de la misma época y de la misma situación. En el fondo, ambas dicen lo mismo" (C. Schmitt, Diálogos, IEP, Madrid, 1962, pp. 86-87).

9. C. Marx y F. Engels, Obras Escogidas, vol. 1, Ed. Progreso, Moscú, 1966, pp. 31-32.

10. Ibídem, p. 39.

11. R. Luxemburg, La revolución rusa, Anagrama, Barcelona, 1975, p. 76. En este escrito, concluido antes de octubre de 1918 , Rosa Luxemburg advierte que "el remedio inventado por Trotsky y Lenin, la supresión de la democracia en general, es aún peor yue el mal que se quiere evitar: sofoca, en efecto, lu fuente viva de la que únicamente pueden surgir las correcciones de las insuficiencias congénitas a las instituciones sociales, una vida política activa, libre y enérgica de las más amplias masas... Lenin y Trotsky, por el contrario, optan por la dietudura en oposición a la democracia y en consecuencia por la dictadura de un puñado de personas..." (Ibidem, pp. 69 y 81 ).

12. J. Stalin y G. Zinoviev, El Gran Debate (1924-26): El socialismo en un solo país (G. Procacci, Ed.), Siglo XXI, Madrid, 1975, p. 114.

13. Ibidem, p. 114.

14. Ibídem, p. 76.

15. Ibídem, p. 115.

16. M. Eastman, The End of Socialism in Russia, Little, Brown \& Company, Boston, 1937. Refiriéndose a intelectuales occidentales como R. Rolland, W. Frank, H. Laski y el matrimonio Wehb, y a revistas liberales como The Nation y The New Republic, Max Eastman escribirá: "It is a strange experience, for one who has lived through these twenty-five years as a Marxian socialist, to see how in proportion as the Soviet regime drops overboard one by one every vestige of socialism, the liheral scholars and litterateurs of the whole world, in so far as they are at all flexible, come over to socialism, and rally with extreme emotion to the defense of the USSR... It is a strange experience, and for one who rests his final hope upon human intelligence, a sad one" (pp. 3-4). Eastman enuncia su tesis con meridiana claridad: "It is the building up of a new privileged caste, and aristocracy of lahor, 
who together with the highly paid foreman and managers can be relied on to support the dictator... The events of August, 1936 (inicio de los procesos de Moscú) are the bloody punctuation of a twelve-year period of counter-revolution. They mean the experiment in socialism in Russia is at an end" (pp. 34 y 46).

17. El escritor francés Albert Camus, en un artículo publicado en 1946, en la revista Combat, con el título "Ni víctimas ni verdugos", refiriéndose al socialismo falseado escribe: "Esta época märea el fin de las ideologías, es decir, de las utopías absolutas que se destruyen a sí mismas, en la historia, por el precio que se acaba pagando por ellas". (A. Camus, Moral y Política, Alianza Ed., Madrid, 1984, p. 84).

La expresión fin de las ideología's se generalizará, a partir de los años cincuenta, en un debate que llega hasta hoy mismo. Véase: C. I. Waxman (Ed.), The End of Ideology Debate, New York, 1968; M. Rejai (Ed.) Decline of Ideology?, Chicago, 1971; y D. Bell, "The End of Ideology Revisited" (Government and Opposition, 2-3, London, Spring-Summer, 1988).

Entre las aportaciones, en lengua españ்$\mathrm{la}$, a la literatura finalista, merecen destacarse: G. Fernández de la Mora, El crepúsculo de las ideologías, Rialp, Madrid, 1965; J. Fueyo, "El fin de la política moderna", ant. cit.; O. Paz, "Fin de un sistema" y "¿Fin de un Imperio?", en Pequeña crónica de grandes días, FCE, México DF, 1990; y R. Cotarelo, "Socialismo y fin del comunismo", en El Basilisco (Oviedo, Primavera, 1991) y "La universalización de la democracia", en Debate Abierto (Madrid, Primavera, 1991).

18. M. Eastman, Stalin's Russia and the Crisis in Socialism, W. W. Norton \& Company, New York, 1939. Véase especialmente el capítulo 3: "Stulin beats Hitler Twenty Ways".

19. M. Eastman, en conversación con Freda Utley y Bertrand Russell en New York City (1941) y en New Leader (August, 4, 1945), cit. en Reflections on the Failure of Socialism, Viewpoint Books, San Diego, California, 1955.

20. M. Eastman, Stalin's Russia... p. 82. Cit. por F. A. Hayek, The Road to Serf- dom (1944) (versión española, Camino de servidumbre, Alianza Ed., Madrid, 1985, p. 55).

21. J. S. Prybyla, "The Road From Socialism: Why, Where, What, and How", en Problems of Communism, Washington DC, January-April, 1991, p.l.

22. F. A. Hayek, The Fatal Conceit: The Errors of Socialism, University of Chicago Press, Chicago, 1.989, pp. 137-138.

23. J. Buchanan, "Socialism Is Dead; Leviathan Lives", conferencia pronunciada en Sidney, Australia (en marzo de 1990) y publicada en The Wall Street Journal, New York, july, 18, 1990.

24. "La evidencia sugiere que el hombre del siglo XX es un neurótico político... Un neurótico político puede ser descrito como alguien que tiene un contacto falso con la realidad, y cuyos juicios están basados, no én hechos, sino en sus propios deseos y temores... El neurótico político lleva su propio Telón de Acero dentro del cráneo." (A. Koestler, en Encounter, London, noviembre de 1953). Según Hayek, "superstición (es) todo" sistema en el que los individuos se imaginan que saben más de lo que en realidad conocen... El liberalismo es, por tanto, científicamente superior al socialismo, $y$ sobre todo al marxismo... que es una superstición... un error cientifico... una vanidad fatal..." (entrevista con Guy Sorman, Los verdaderos pensadores de nuestro tiempo, Seix Barral, Barcelona, 1991, p. 189). En la misma línea, Karl Popper sostiene que los enemigos de la sociedad abierta son "los fabricantes de ideologías, los impostores... desgraciadamente dominan las ciencias humanas... El bluff ha reemplazado a la inteligencia de los hechos, y los intelectuales se han precipitado por esta brecha. Junto a Hegel, Marx instauró en los tiempos modernos el culto a las ideas abstractas: la religión del Estado, de la Nación, del Proletariado... El éxito de estas ideologías proviene de que eximen de reflexionar" (entrevista con Guy Sorman, ob. cit., p. 253).

25. R. Cotarelo, La izquierda: desengaño, resignación y utopía, Ed. Drac, Barcelona, 1989 , p. 11. Nada ilustra mejor estas palabras que contemplar la escena política internacional y española. En la presentación 
del libro del profesor Cotarelo, Fernando Claudín, prestigioso escritor de izquierdas hoy lamentablemente desaparecido, ex-comunista y gran conocedor de la historia del pensamiento socialista-comunista, tuvo la honestidad intelectual de reconocer que el fin del comunismo en Europa necesariamente afectará (intelectual y políticamente) a toda la izquierda, incluido el socialismo democrático. Esto, dicho en 1989, significa que la socialdemocracia occidental (revisionista y reformista) desde 1917 no ha llegado todavía al fondo de la cuestión.

26. J. Prybyla, ob. cit., p. 2

27. A. Touraine, El postsocialismo (1980), Planeta, Barcelona, 1982; del mismo, "El nacimiento de las sociedades post-comunistas", en la revista Claves, $\mathrm{n}^{2}$ 11, Madrid, abril de 1991; Z. Brzezinski, The Grand Failure (The Birth and Death of Communism in the Twentieth Century) (1980), Macmillan Pub. Company, New York, 1990; del mismo, "Toward A Common European Home", en Problems of Communism, Washington DC, November-December 1989, y "Beyond Chaos. A Policy for the West", en The National Interest, N. Y., Spring 1990; J. Prybyla, "The Road From Socialism", ant. cit.

28. Z. Brzezinski, The Grand Pailure, ant. cit., p, 255.

29. R. F. Staar, "Checklist of Communist Parties in 1989", en Problems of Communism, Washington DC, March-April 1990.

30. J. Ortega y Gasset, "Imperativo de la intelectualidad", en la revista España, Madrid, 1922; J. Benda, La traición de los intelectuales (1927), FC ed., Buenos Aires, 1974.

31. R. Aron, El opio de los intelectuales (1955), Ed. Siglo Veinte, Buenos Aires, 1967. Este gran escritor político francés hizo también su aportación a la literatura finalista (fin de las ideologías, fin del socialismo) a principios de los años cincuenta: R. Aron, "The End of the Socialist Myth" (1951), en su libro (versión inglesa) The Century of Total War, The Beacon Press, Boston, 1955.

32. J. F. Revel, La tentación totalitaria (1976), Plaza y Janés, Barcelona, 1976, pp. 87 y ss. Revel citu una preciosa frase de Roger Garaudy en 1965: "Vamos hacia el socialismo con el Corán en una mano y $E \boldsymbol{l}$ Capital en la otra." (p. 104). Se trata, sin duda, de un intelectual emblemático, ya que previamente, siendo militante comunista, había abogado por el "diálogo marxista-cristiano", e, incluso, hizo sus pinitos como teólogo de la muerte de Dios (Véase su libro Dieu est mort. Etude sur Hegel, PUF, París, 1962).

33. Cit. por J. Prybyla, ob. cit., p. 7. János Kornai, economista húngaro y que sabe de lo que está hablando, es autor de algunos trabajos fundamentales, desde la perspectiva económica, para una sociología de la transición del comunismo al capitalismo: "Hungarian Reform Process", en V. Nee y D. Stark (Eds.), Remaking the Economic Institutions of Socialism, Stanford UP, Stanford, California, 1989; y The Road to a Free Economy. Shifting from a Socialist System: The Example of Hungary, W. W. Norton, New York, 1990. 